OPEN ACCESS

Edited by:

Weiren Zhu,

Shanghai Jiao Tong University, China

Reviewed by:

Yongjun Huang,

University of Electronic Science and

Technology of China, China

Qian Sun,

Nankai University, China

*Correspondence:

Jun Yang

junyang@hfut.edu.cn

Specialty section:

This article was submitted to

Optics and Photonics,

a section of the journal

Frontiers in Physics

Received: 23 September 2020

Accepted: 05 November 2020

Published: 25 November 2020

Citation:

Deng G, Lv K, Sun H, Yin Z and Yang J (2020) Stereo Perfect Metamaterial Absorber Based on Standing Gear-

Shaped Resonant Structure With

Wide-Incident-Angle Stability.

Front. Phys. 8:609527.

doi: 10.3389/fphy.2020.609527

\section{Stereo Perfect Metamaterial Absorber Based on Standing Gear-Shaped Resonant Structure With Wide-Incident-Angle Stability}

\author{
Guangsheng Deng, Kun Lv, Hanxiao Sun, Zhiping Yin and Jun Yang* \\ Special Display and Imaging Technology Innovation Center of Anhui Province, Academy of Opto-Electronic Technology, Hefei \\ University of Technology, Hefei, China
}

In this work, a single-band metamaterial absorber (MA) based on a three dimensional (3D) resonant structure is presented. The unit cell is composed of a standing gear-shaped resonator, which is embedded in the dielectric substrate. The proposed 3D MA is ultrathin with a total thickness of $2.3 \mathrm{~mm}$, corresponding $0.077 \lambda_{0}$ at its center frequency. The simulation results demonstrate a high absorption peak at $10.1 \mathrm{GHz}$ with absorptivity of 99.9\%. The proposed 3D MA is insensitive to the polarization of the incident wave due to its rotationally symmetric structure. Moreover, the proposed 3D MA exhibits a wide-incidentangle stability, as absorptivity of more than $85 \%$ can be achieved for both TE and TM incidences with incident angle up to $60^{\circ}$. Most importantly, multiband electromagnetic wave absorption of the stereo MA can be enabled by adjusting the structural parameters of the standing gear. The proposed structure is compatible with 3D printing technology and has potential applications in electromagnetic shielding.

Keywords: metamaterial, absorber, three dimension, single-band, wide-incident-angle stability

\section{INTRODUCTION}

Electromagnetic (EM) metamaterials that consist meta-molecules arranged in an array of subwavelength pe have attracted intense attention due to their unique properties, such as negative refractive index [1] and inverse Doppler effects [2]. Recently, the perfect absorber has been closely related to metamaterials, which has potential applications in biological sciences [3], sensing $[4,5]$, communications $[6,7]$ and solar energy harvesting [8].

The perfect metamaterial absorber (MA) was firstly presented by Landy et al. [9]. Since then the MAs have been designed to exhibit different characteristics such as single-band [10, 11], dual-band $[12,13]$, multiband absorption [14-17] and broadband absorption [18-21]. Till now, most of the reported MAs are based on planar resonators (2D structure) [11, 22-24]. However, the absorbing performance of the planar design will deteriorate significantly under oblique incidences with large incident angles.

Recently, the 3D printing technology [25] has experienced significant development and paved a way for the design and fabrication of 3D structures. One of the advantages of the 3D design scheme is that it can improve the design flexibility of the MA by providing additional freedom in structure design. Hence a 3D MA can enable higher absorption at oblique incidences. For instance, Wu et al. presented a symmetric all-metal three-dimensional (3D) MA by using two orthogonally oriented copper stand-up spilt ring resonators at $\mathrm{THz}$ frequency and obtained more than $90 \% \mathrm{THz}$ wave 
copper

\section{A}

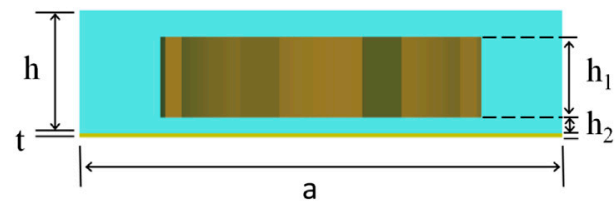

C

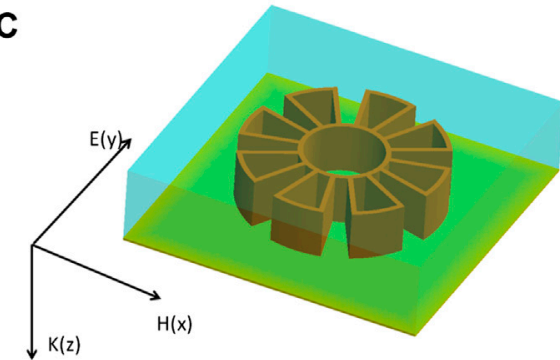

conductive silver paste

B

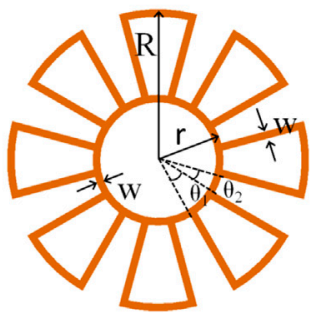

FIGURE 1 | Schematic geometry of a unit cell for the proposed 3D MA (A) Front view (B) layout of the gear-shaped resonator, and (C) perspective view of the unit cell.

absorption for both TE and TM polarization with incident angle up to $60^{\circ}$ [26]. Lv et al. [27] proposed a three-dimensional ultrabroadband metamaterial absorber with full graphite structure that exhibited excellent absorption properties at large incident angles.

In this paper, a novel single-band and polarization-insensitive MA based on a 3D gear-shaped resonant structure is presented and near unity (99.9\%) absorption at absorption peak frequency of $10.1 \mathrm{GHz}$ under normal incidence is numerically demonstrated. Here, we introduce a new strategy on the design of 3D MAs by extruding planar resonator along its normal direction. Compared with the original planar resonant structure, the proposed structure can offer higher absorption for both TE and TM-polarized waves with large incident angles. The surface current and the field density distributions are investigated to explore the absorption mechanism of the $3 \mathrm{D}$ structure. Moreover, by adjusting the number of gear teeth, perfect single- or multi-band absorption can be achieved based on the stereo resonant structure. The proposed MA has the potential to be applied in the energy-harvesting and EM shielding applications.

\section{STRUCTURE DESIGN AND SIMULATION}

Figure 1 shows the geometry of a unit cell of the proposed singleband 3D MA. The standing gear-shaped 3D resonator made of silver ink with an electrical conductivity of $\sigma=5.88 \times 10^{5} \mathrm{~S} / \mathrm{m}$, is embedded in the dielectric substrate. Here, we select silver ink to construct metallic standing gear, as it is perfectly compatible with $3 \mathrm{D}$ printing technology. The substrate is realized on photosensitive resin with a relative permittivity of 2.9 and a loss tangent of 0.02. Moreover, in order to eliminate the EM wave transmission through the structure, a $0.017 \mathrm{~mm}$ thick copper
TABLE 1 | Dimensions and parameters of the proposed 3D MA.

Parameter Value $(\mathrm{mm})$ Parameter Value $(\mathrm{mm})$ Parameter Value $(\mathrm{mm})$

$\begin{array}{cccccc}a & 9 & h & 2.3 & w & 0.15 \\ R & 3 & h_{1} & 1.5 & \theta_{1} & 30^{\circ} \\ r & 1.3 & h_{2} & 0.1 & \theta_{2} & 15^{\circ}\end{array}$

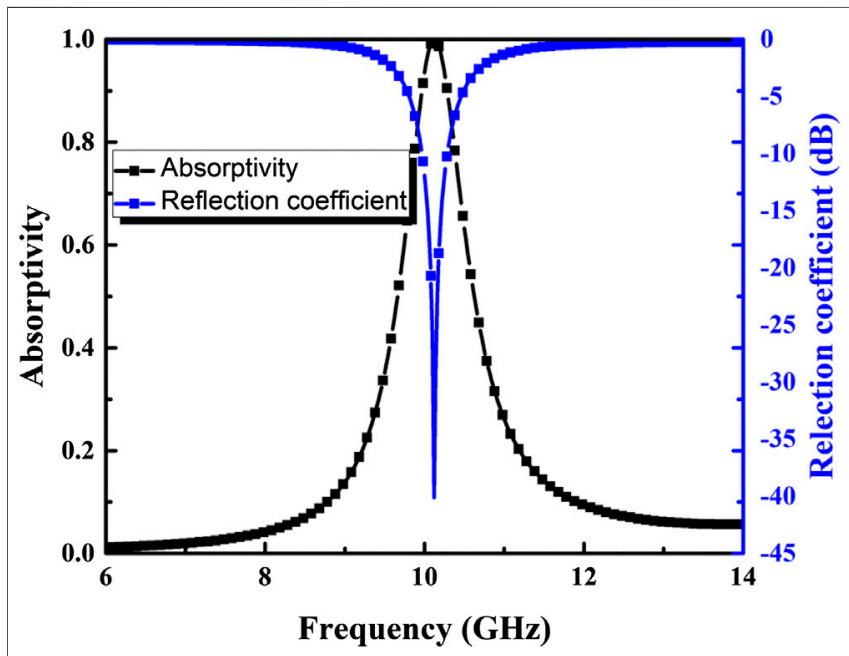

FIGURE 2 | Absorption and reflection spectrum of the proposed MA under normal incidence.

plate with an electric conductivity of $5.8 \times 10^{7} \mathrm{~S} / \mathrm{m}$ is covered on the bottom of the structure as a ground plane. Figure 1 shows the configuration of the unit cell structure, while the optimized parameters of the 3D MA are listed in Table 1. The simulation results were obtained using a finite-element method 

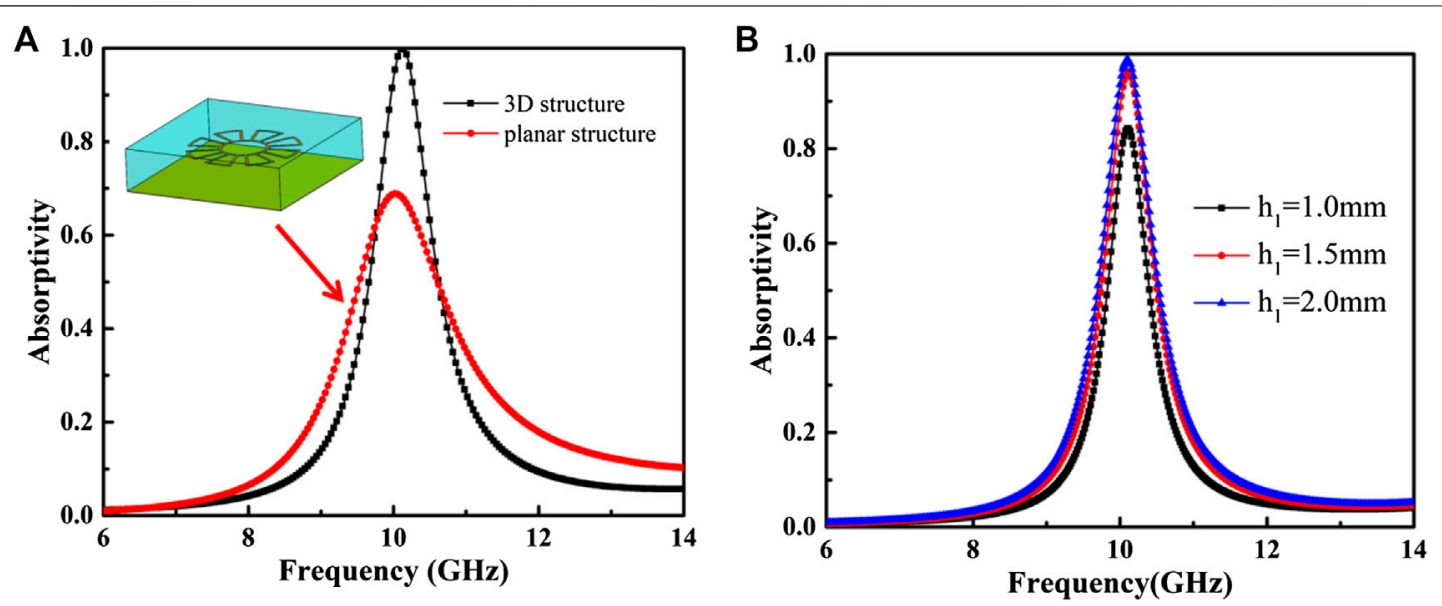

FIGURE 3 | (A) Comparison of the absorption spectrum of the proposed 3D MA and the planar design under normal incidence (B) Absorption spectrum dependency on the thickness of the standing gear-shaped structure at incidence angle of $45^{\circ}$.

A

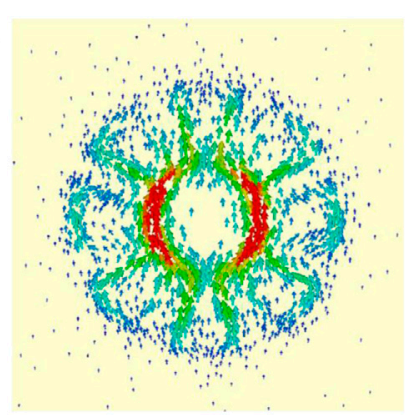

C

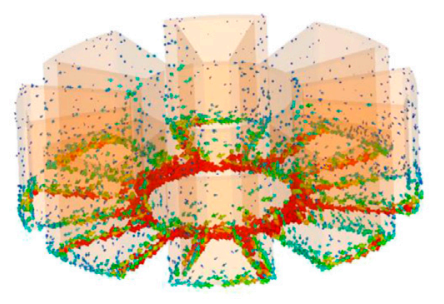

B

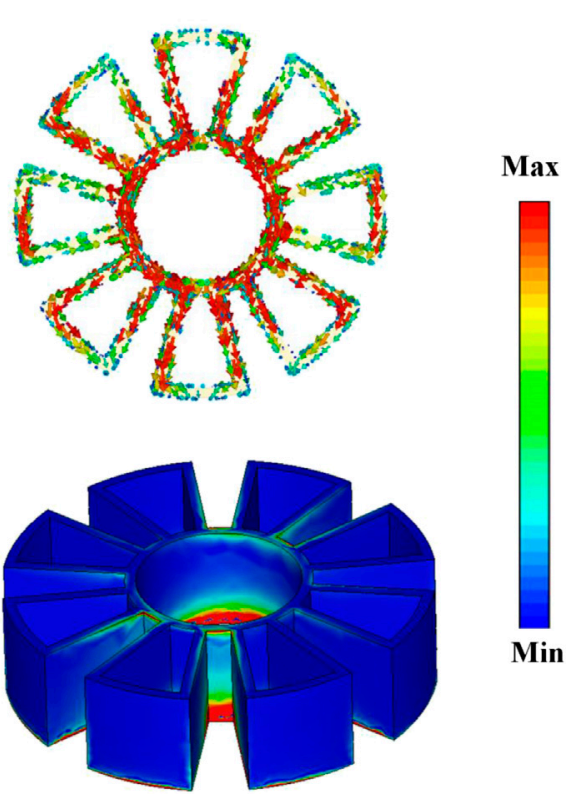

FIGURE 4 | Simulated surface current distribution on (A) bottom ground layer (B) 3D resonator on top view, and (C) 3D resonator on perspective view, and (D) distribution of the power loss on the 3D resonator.

A

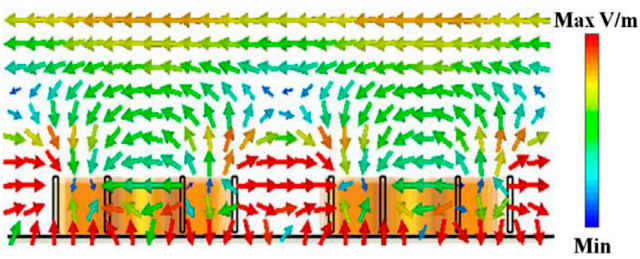

B

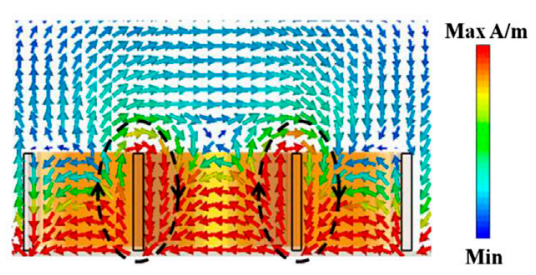

FIGURE 5 | Simulated (A) electric, and (B) magnetic field distributions within the 3D MA. 

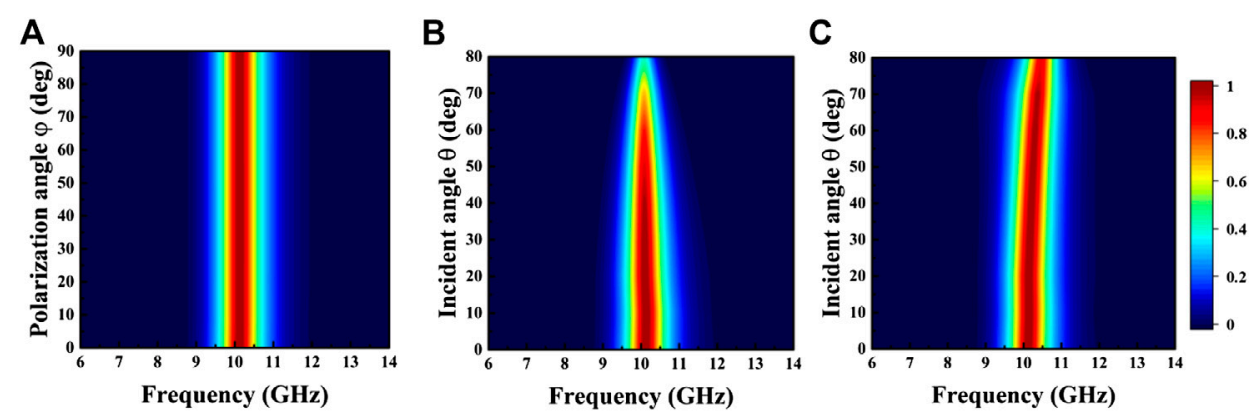

FIGURE 6 | Absorption spectra for (A) different polarization angles $\varphi$ and different incidence angles $\theta$ for (B) TE, and (C) TM polarization.

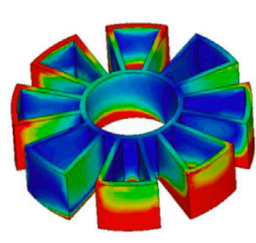

$\theta=0^{\circ}$

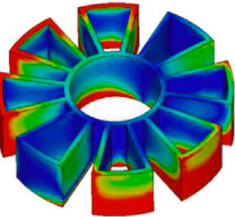

$\theta=30^{\circ}$

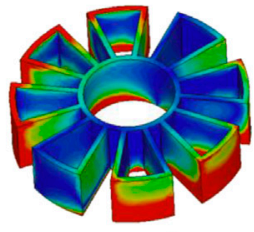

$\theta=60^{\circ}$

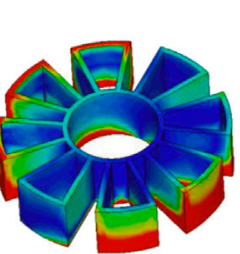

$\theta=75^{\circ}$

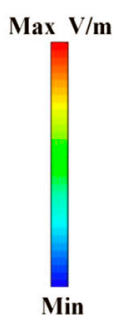

FIGURE 7 | Simulated electric field distributions for TM polarization under different incident angles $\theta$.

(FEM). In the simulation, the unit cell boundary conditions were applied in $x$ and $y$ directions, and the open space boundary condition was utilized in the $z$ direction. The absorptivity (A) can be defined as $A(\omega)=1-R(\omega)-T(\omega)=1-\left|S_{11}(\omega)\right|^{2}-\left|S_{21}(\omega)\right|^{2}$, where $S_{11}(\omega)$ and $S_{21}(\omega)$ are the reflection and the transmission coefficients, respectively. The transmission coefficient $S_{21}(\omega)$ is zero due to the existence of copper ground. Therefore, the absorptivity can be simplified as $A(\omega)=1-\left|S_{11}(\omega)\right|^{2}$.

The simulated absorption spectrum of the proposed 3D MA under normal incidence is shown in Figure 2. It can be seen from Figure 2 that there exists a sharp absorption peak located at $10.1 \mathrm{GHz}$ with an absorptivity of $99.9 \%$. Hence, the proposed MA exhibits perfect single band incident electromagnetic wave absorption.

\section{RESULTS AND DISCUSSION}

Figure $3 \mathbf{A}$ compares the absorptivity of the proposed stereo structure and the planar design under normal incidence. It can be seen from the figure that the absorptivity of the stereo MA is much larger than that of the planar structure at peak resonant frequency. In order to analyze the dependence of the height of the stereo resonant structure $\left(h_{1}\right)$ on the EM wave absorption under oblique incidence, the influence of $h_{1}$ on absorption spectrum at wave incident angle of $45^{\circ}$ is simulated and the results are shown in Figure 3B. From Figure 3B, the absorptivity increases till a nearly perfect absorption with the increase of $h_{1}$, which demonstrates the outstanding absorbing performance of the proposed stereo structure at wide incidence angles.
The surface current distribution on the gear-shaped $3 \mathrm{D}$ resonator and the copper ground under the TM-polarized incidence at peak resonant frequency of $10.1 \mathrm{GHz}$ are depicted in Figure 4. It can be seen from Figure 4A that the surface current on the stereo structure flows in the same direction along the E-field vector of the incident wave, where the electric resonance can be excited within the MA. Meanwhile, Figure 4B shows that the current is mainly concentrated in the inner ring of the standing gear-shaped structure, and the current flow direction in the copper ground is in reverse with that in the stereo resonant structure, as the anti-parallel current will lead to a magnetic resonance. Hence, both magnetic and electric resonances are responsible for EM wave absorption under normal incidence. Figure 4C illustrates the perspective view of the current distribution on the stereo gear-shaped resonator. Although the surface current is strongly concentrated in the bottom of the gear, the current flow on the side wall of the standing sectors also contributes the power consumption as shown in Figure 4C. The power loss distribution shown in Figure 4D confirms that the extra power consumption on the standing walls is responsible for the stronger absorption compared with planar structure. Moreover, from Figure 4D, one can anticipate more power dissipation on the walls by increasing the height of the standing gear, which is in accordance with our previous discussion of the absorption dependence on gear height $h_{1}$.

Figure 5 shows the electric and magnetic field distributions to further explain the mechanism of the enhanced absorption of the stereo structure. It can be seen from the electric field distribution shown in Figure 5A that the strong coupling between adjacent 


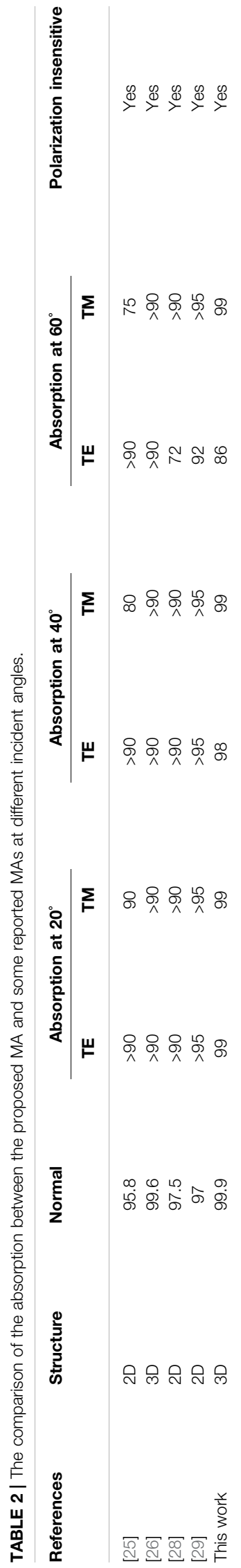

standing elements will enhance the intensity of electric resonance within the structure. Moreover, the magnetic field distribution illustrated in Figure 5B suggests that there exist two pairs of codirectional magnetic field rings due to the introduction of the standing walls. Since the magnetic rings can be regarded as an electric dipole, the oscillation of the dipole results in a strong electric response of the MA which further enhances the EM wave absorption.

\section{ABSORPTION SPECTRUM DEPENDENCE ON WAVE POLARIZATION AND INCIDENT ANGLE}

In many practical applications, the characteristic of polarizationindependent is an important criterion to evaluate the absorption performance of the MAs. Figure 6A illustrates the simulated absorption maps of the proposed MA under different polarization angles. It can be observed that the absorption of the MA is insensitive to different polarization angles, which is attributed to the rotationally symmetric layout of the cell structure.

The absorbing performance of the stereo MA is further investigated for TE- and TM-polarized incidences at different incident angles, and the simulated results are shown in Figures 6B,C, respectively. For the TE-polarized incidence, the absorptivity remains above $85 \%$ with incident angle $\theta$ up to $60^{\circ}$. However, the further increase of $\theta$ leads to a dramatically degradation of EM wave absorption. For the TM-polarized incidence, the absorptivity remains greater than $90 \%$ when incident angle $\theta$ reaches up to $80^{\circ}$. However, the absorption peak frequency slightly blue-shifted with the increase of $\theta$. Hence, the proposed structure exhibits wide-incident-angle stability for both TE and TM polarizations. In order to gain the electromagnetic response of the stereo structure under oblique incidences, the electric field distributions for TMpolarized incidence with incident angle $\theta=0^{\circ}, 30^{\circ}, 60^{\circ}$ and $75^{\circ}$ are shown in Figure 7. It can be seen from the figure that both the distribution and the intensity of the electric field are insensitive to wave incident angle. Thus, the proposed stereo MA has a good capacity on effective EM wave absorption at wide incident angles. The equivalent circuit model for analysis of the absorption peaks under oblique incidence is not given in this paper. However, the mechanism can be briefly illustrated as follow: the impedance matching of free space and the input impedance of the stereo structure, which determines the absorptivity, is strongly dependent on the EM wave incident angle. Compared with the planar structure, the standing walls of the gear enables an extra compensation of impedance matching at wide incident angles, hence enhancing the wave absorption. In Table 2, we compared the performance of the proposed MA with some reported MAs at different incident angles. As it can be seen that our proposed structure exhibits stronger incident wave absorption under oblique incidences for both TE and TM polarization.

Another advantage of the proposed standing gear-shaped resonant structure is the adjustable perfect single- or multiband absorption. In general, by increasing the amount of the 


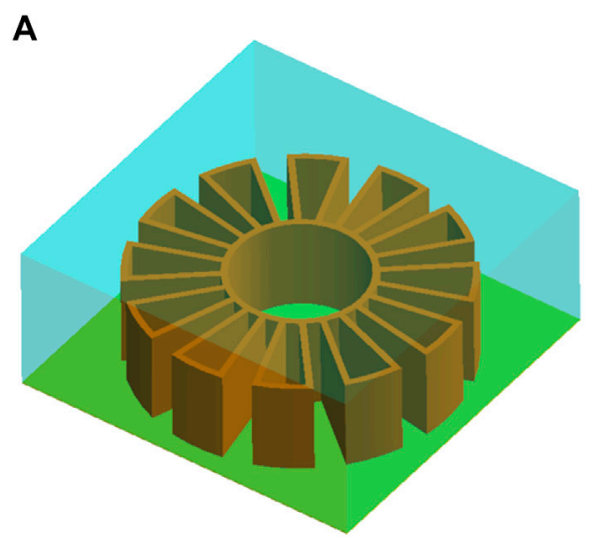

B

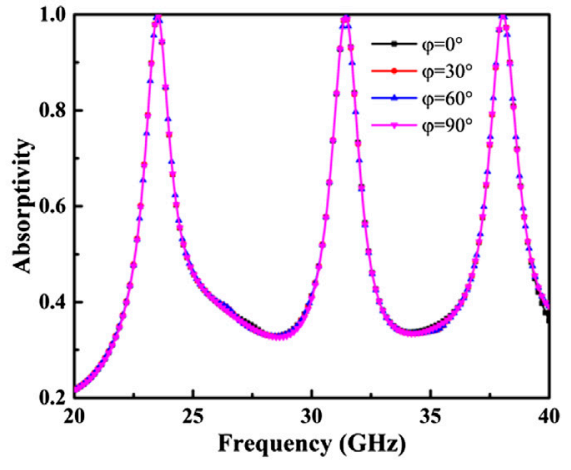

FIGURE 8 | (A) Configuration of the triple-band 3D MA (B) Simulated absorption spectrum under normal incidence with different polarization angles $\varphi$.

TABLE 3 | Dimensions and parameters of the triple-band 3D MA.

\begin{tabular}{lcccc}
\hline Parameter & Value $(\mathbf{m m})$ & Parameter & Value $(\mathbf{m m})$ & Value $(\mathbf{m m})$ \\
\hline$A$ & 8 & $h$ & 3.4 & $W$ \\
$R$ & 3.6 & $h_{1}$ & 2.1 & 0.15 \\
$R$ & 1.6 & $h_{2}$ & 0 & $\theta_{1}$ \\
\hline
\end{tabular}
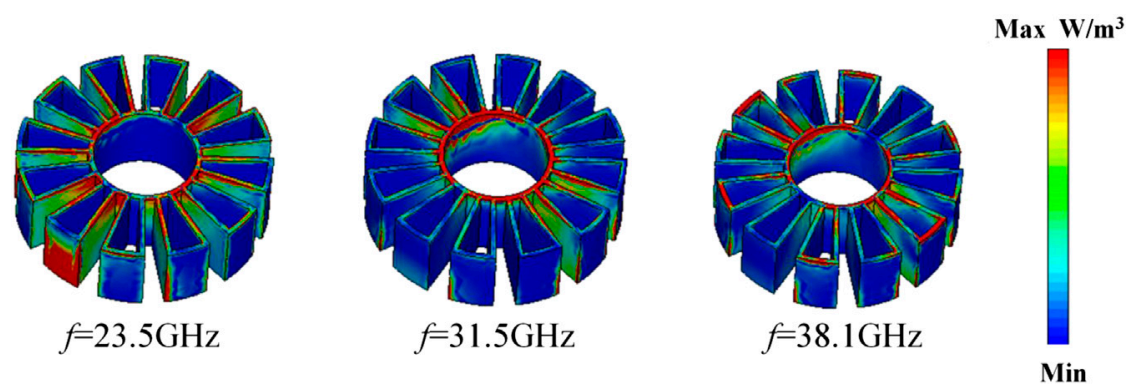

FIGURE 9 | Power loss density distributions on the 3D resonator at three peak absorption frequencies.

gear teeth, the resonant modes within the structure will also increase due to the enhanced coupling between these adjacent teeth. Hence, one can anticipate more absorption peaks with lager counts of gear teeth. For instance, a perfect triple-band absorption can be achieved by optimizing the structural parameters of the stereo structure. The layout and the absorption spectrum of the triple-band MA are illustrated in Figure 8, while the detailed parameter dimensions of the unit cell structure are listed in Table 3 . The simulation results demonstrate three distinct absorption peaks at 23.5, 31.5, and $38.1 \mathrm{GHz}$ with absorption of $99.9,99.5$, and $99.9 \%$, respectively. Figure 9 shows the power loss density distributions of the stereo triple-band MA for TE-polarized incidence at three resonant frequencies. It can be seen from Figure 9 that the incident power is significantly consumed at the side wall and the top plane of the standing gear-shaped resonator for these three resonant peaks, which is different from the absorption mechanism discussed earlier in the article. Hence, the stereo structure provides more degree of freedom in constructing multi-functional MAs compared with the planar design.

\section{CONCLUSION}

A standing gear-shaped perfect metamaterial absorber with wideincident-angle stability is presented in this paper. The stereo resonator that is constructed with conductive silver ink, can be embedded into the substrate using $3 \mathrm{D}$ printing technology. The rotationally symmetric structure enables the polarizationindependent absorption of the proposed MA under normal incidence, while the stereo resonator also endows the MA with good absorbing capacity at wide incident angles. The simulation results demonstrate and validate that the proposed MA exhibits near unity single-band absorption under normal incidence. Moreover, for oblique incidences, the proposed MA maintains 
absorptivity above 85 and $95 \%$ up to incident angles of $60^{\circ}$ and $80^{\circ}$ for TE and TM polarizations, respectively. In addition, a perfect multi-band absorption can be achieved by adjusting the structural parameters of the stereo structure. The stereo absorber has potential applications in the energy harvesting and stealth fields.

\section{DATA AVAILABILITY STATEMENT}

The raw data supporting the conclusions of this article will be made available by the authors, without undue reservation.

\section{REFERENCES}

1. Smith DR, Pendry JB, Wiltshire MCK. Metamaterials and negative refractive index. Science (2004) 305:788-92. doi:10.1126/science.1096796

2. Seddon N, Bearpark T. Observation of the inverse Doppler effect. Science (2003) 302:1537-40. doi:10.1126/science.1089342

3. Marin BC, Ramirez J, Root SE, Aklile E, Lipomi DJ. Metallic nanoislands on graphene: a metamaterial for chemical, mechanical, optical, and biological applications. Nanoscale Horizons (2017) 2:311-8. doi:10.1039/c7nh00095b

4. Yi Z, Huang J, Cen CL, Chen XF, Zhou ZG, Tang YJ, et al. Nanoribbon-ring cross perfect metamaterial graphene multi-band absorber in $\mathrm{THz}$ range and the sensing application. Results Phys (2019) 14:102367. doi:10.1016/j.rinp.2019. 102367

5. Sang T, Wang R, Li JL, Zhou JY, Wang YK. Approaching total absorption of graphene strips using a c-Si subwavelength periodic membrane. Optic Commun (2018) 413:255-60. doi:10.1016/j.optcom.2017.12.065

6. Yang W, Lin YS. Tunable metamaterial filter for optical communication in the terahertz frequency range. Optics Express (2020) 28: 17620-9. doi:10.1364/Oe. 396620

7. Yu BY, Zhao YJ, Chen JQ, Ge Y, Chen XF. Broadband transparent metamaterial absorber in wireless communication band based on indium tin oxide film. Int J RF Microw Comput-Aid Eng (2019) 29:e219551. doi:10. $1002 /$ mmce. 21955

8. Hossain MJ, Faruque MRI, Islam MT. Perfect metamaterial absorber with high fractional bandwidth for solar energy harvesting. PLoS ONE (2018) 13: e0207314. doi:10.1371/journal.pone.0207314

9. Landy NI, Sajuyigbe S, Mock JJ, Smith DR, Padilla WJ. Perfect metamaterial absorber. Phys Rev Lett (2008) 100:279-82. doi:10.1103/PhysRevLett.100.207402

10. Cheng YZ, Yang HL, Cheng ZZ, Wu N. Perfect metamaterial absorber based on a split-ring-cross resonator. Appl Phys Mater Sci Process (2011) 102:99-103. doi:10.1007/s00339-010-6022-4

11. Lim D, Lee D, Lim S. Angle- and polarization-insensitive metamaterial absorber using Via array. Sci Rep (2016) 6:39686. doi:10.1038/srep39686

12. Wang BX, He YH, Xu NX, Wang XY, Wang YC, Cao JJ. Design of dual-band polarization controllable metamaterial absorber at terahertz frequency. Results Phys (2020) 17:103077. doi:10.1016/j.rinp.2020.103077

13. Wang CC, Huang $\mathrm{MH}$, Zhang $\mathrm{Z}$, Xu W. Dual band metamaterial absorber: combination of plasmon and Mie resonances. J Mater Sci Technol (2020) 53: 37-40. doi:10.1016/j.jmst.2020.02.058

14. Cheng YZ, Cheng ZZ, Mao XS, Gong RZ. Ultra-thin multi-band polarizationinsensitive microwave metamaterial absorber based on multiple-order responses using a single resonator structure. Materials (2017) 10:1241. doi:10.3390/ma10111241

15. Tran CM, Pham HV, Nguyen HT, Nguyen TT, Vu D, Do TH. Creating multiband and broadband metamaterial absorber by multiporous square layer structure. Plasmonics (2019) 14:1587-92. doi:10.1007/s11468-019-00953-6

16. Wang BX, Wang GZ, Sang T, Wang LL. Six-band terahertz metamaterial absorber based on the combination of multiple-order responses of metallic patches in a dual-layer stacked resonance structure. Sci Rep (2017) 7:41373. doi:10.1038/srep41373

17. Zhao Y, Fu CJ. Design of multiband selective near-perfect metamaterial absorbers with $\mathrm{SiO}_{2}$ cylinder/rectangle shell horizontally embedded in

\section{AUTHOR CONTRIBUTIONS}

GD conceived the research, wrote the manuscript; KL and HS conducted simulations and analysis, ZY and JY conducted analysis; KL wrote the manuscript.

\section{FUNDING}

This research was funded by National Natural Science Foundation of China (61871171).

opaque silver substrate. Int J Heat Mass Tran. (2017) 113:281-5. doi:10. 1016/j.ijheatmasstransfer.2017.05.090

18. Yu P, Besteiro LV, Huang YJ, Wu J, Fu L, Tan HH, et al. Broadband metamaterial absorbers. Adv Opt Mater (2019) 7:1800995. doi:10.1002/ adom.201800995

19. Zou JH, Yu P, Wang WH, Tong X, Chang L, Wu C. Broadband mid-infrared perfect absorber using fractal Gosper curve. J Phys D Appl Phys (2020) 53: 10516. doi:10.1088/1361-6463/ab57ea

20. Du XM, Yan FP, Wang W, Tan SY, Zhang LN, Bai ZY, et al. A polarizationand angle-insensitive broadband tunable metamaterial absorber using patterned graphene resonators in the terahertz band. Optic Laser Technol (2020) 132:106513. doi:10.1016/j.optlastec.2020.106513

21. Akafzade H, Sharma SC. New metamaterial as a broadband absorber of sunlight with extremely high absorption efficiency. AIP Adv (2020) 10: 035209. doi:10.1063/1.5131630

22. Lee D, Hwang JG, Lim D, Hara T, Lim S. Incident angle- and polarizationinsensitive metamaterial absorber using circular sectors. Sci Rep (2016) 6 27155. doi:10.1038/srep27155

23. Huang YJ, Wen GJ, Zhu WR, Li J, Si LM, Premaratne M. Experimental demonstration of a magnetically tunable ferrite based metamaterial absorber. Optics Express (2014) 22:16408-17. doi:10.1364/Oe.22.016408

24. Wu KM, Huang YJ, Wanghuang TL, Chen WJ, Wen GJ. Numerical and theoretical analysis on the absorption properties of metasurface-based terahertz absorbers with different thicknesses. Appl Opt (2015) 54:299-305. doi:10.1364/Ao.54.000299

25. Lu YJ, Chi BH, Liu DY, Gao S, Gao P, Huang Y, et al. Wideband metamaterial absorbers based on conductive plastic with additive manufacturing technology. ACS Omega (2018) 3:11144-50. doi:10.1021/acsomega.8b01223

26. Wu M, Zhao XG, Zhang JD, Schalch J, Duan GW, Cremin K, et al. A threedimensional all-metal terahertz metamaterial perfect absorber. Appl Phys Lett (2017) 111:051101. doi:10.1063/1.4996897

27. Lv F, Xiao ZY, Lu XJ, Chen MM. Three-dimensional ultra-broadband metamaterial absorber with full graphite structure. J Electron Mater (2020) 49:689-94. doi:10.1007/s11664-019-07735-0

28. Yoo M, Kim HK, Lim S. Angular- and polarization-insensitive metamaterial absorber using subwavelength unit cell in multilayer technology. IEEE Antenn Wireless Propag Lett (2016) 15:414-7. doi:10.1109/Lawp.2015. 2448720

29. Zhu B, Wang Z, Huang C, Feng Y, Zhao J, Jiang T. Polarization insensitive metamaterial absorber with wide incident angle. Prog Electromagn Res Pier (2010) 101:231-9. doi:10.2528/Pier10011110

Conflict of Interest: The authors declare that the research was conducted in the absence of any commercial or financial relationships that could be construed as a potential conflict of interest.

Copyright (c) 2020 Deng, Lv, Sun, Yin and Yang. This is an open-access article distributed under the terms of the Creative Commons Attribution License (CC BY). The use, distribution or reproduction in other forums is permitted, provided the original author(s) and the copyright owner(s) are credited and that the original publication in this journal is cited, in accordance with accepted academic practice. No use, distribution or reproduction is permitted which does not comply with these terms. 